\title{
Quantized Passive Dynamic Output Feedback Control with Actuator Failure
}

\author{
Zu-Xin Li, ${ }^{1,2}$ Ke-Xia Zhou, ${ }^{2}$ Yan-Feng Wang, ${ }^{1}$ Hui-Ying Chen, ${ }^{1}$ and Yi Qian ${ }^{1}$ \\ ${ }^{1}$ School of Engineering, Huzhou University, Huzhou, Zhejiang 313000, China \\ ${ }^{2}$ The Key Lab for IOT and Information Fusion Technology of Zhejiang, The Institute of Information and Control, \\ Hangzhou Dianzi University, Hangzhou 310018, China \\ Correspondence should be addressed to Zu-Xin Li; lzx@zjhu.edu.cn
}

Received 27 July 2016; Accepted 9 October 2016

Academic Editor: Yakov Strelniker

Copyright (C) $2016 \mathrm{Zu}$-Xin Li et al. This is an open access article distributed under the Creative Commons Attribution License, which permits unrestricted use, distribution, and reproduction in any medium, provided the original work is properly cited.

\begin{abstract}
This paper investigates the problem of passive dynamic output feedback control for fuzzy discrete nonlinear systems with quantization and actuator failures, where the measurement output of the system is quantized by a logarithmic quantizer before being transferred to the fuzzy controller. By employing the fuzzy-basis-dependent Lyapunov function, sufficient condition is established to guarantee the closed-loop system to be mean-square stable and the prescribed passive performance. Based on the sufficient condition, the fuzzy dynamic output feedback controller is proposed for maintaining acceptable performance levels in the case of actuator failures and quantization effects. Finally, a numerical example is given to show the usefulness of the proposed method.
\end{abstract}

\section{Introduction}

History has witnessed the development of fuzzy logic theory, and it has been demonstrated to be effective in handling complex nonlinear problems. Wherein, as a common typical model, $T$-S fuzzy model is often used for stability analysis and controller design [1]. Fuzzy model has provided a way to approximate nonlinear system so that some linear system methods can be used for the analysis of nonlinear systems [2-4]. In the past few decades, many important results have been reported. For instance, the problems of stability and stabilization of fuzzy systems have been investigated in [58] and results on filter and controller designs with $H_{\infty}$ performance have been reported in [9-14].

Modern control systems have been becoming increasingly complex, which cause researcher's attention to focus on the system reliability and security. Reliable control is in the process of controller design, and the system components (such as actuators and sensors) failure has been considered. So no matter what kind of failure occurs, it can ensure that the system is stable and the performance is within the acceptable range. The design of conventional control system is usually assumed that the system components are in good condition (i.e., without any fault); however, due to the natural aging of the components, harsh environmental changes, and other factors, the actuator inevitably fails, which will not only worsen the whole system performance but also even lead to the system instability. Therefore, when the components of control system fail, how to design an effective controller or a reliable filter to improve reliability of system is of profound significance in both theoretical research and practical engineering [15-19]. In [15], a reliable filter is designed to ensure a strictly dissipative performance for the filtering error system by solving a convex optimization problem. In [17], sensor fault estimation and fault-tolerant control for Markovian jump systems are investigated. The problems of $H_{\infty}$ and passive filtering of singular system with sensor fault are concerned in [18].

NCSs have been successfully applied in a broad range of areas since they possess many advantages including flexibility in system implementation, reducing cost of installation, and facilitating system maintenance [20-24]. However, the characteristic of the shared network always causes some problems. One of them is packet dropouts, which degrades the system performance and even makes the system unstable. Another problem is the limited communication capacity which causes many researchers to focus on the problem of quantization. In [25-27], the state feedback controller is 
designed for nonlinear system. However, the drawback of the state feedback controller is that full knowledge of the state vector is usually unavailable. Moreover, the dynamic output feedback controller design has been investigated [28, 29]. In contrast to the existing results, the combination of a fuzzy-basis-dependent Lyapunov function approach and transformation on the controller parameters is used which lead to a less conservative result. Moreover, the consideration of both quantization and actuator failures further reduces the conservatism. To the best of the authors' knowledge, the dynamic output feedback control with quantization and actuator failures has not been fully investigated, which motivates the research of this article.

This paper considers the quantized passive output feedback control for the fuzzy discrete nonlinear systems with actuator failures. Sufficient conditions are derived to guarantee that the closed-loop system is mean-square stable and satisfies the prescribed passive performance. The dynamic output feedback controller is taken into account, which avoids the case where the state is unmeasured. The main contributions can be summarized as follows: (1) the fuzzy dynamic output feedback controller is proposed for minimizing the system normal level and maintaining acceptable levels in the case of actuator failures, since it often does not wish to sacrifice the normal performance for the infrequent faulty cases; (2) with the consideration of actuator failures, the robust passive dynamic output feedback controllers are designed to deal with the measurement losses and mitigate quantization effects.

The rest of this paper is organized as follows. Section 2 formulates the problem. The stability condition and passive performance of the closed-loop system are given in Section 3. The dynamic output feedback controller is proposed in Section 4. An illustrated example is given to demonstrate the effectiveness of the proposed method in Section 5 and the conclusion is presented in Section 6.

Notation. In this paper, real numbers, Euclidean space with $n$ dimension, and $n \times m$ real matrices are denoted by $\mathbb{R}, \mathbb{R}^{n}$, and $\mathbb{R}^{n \times m}$, respectively. $M^{T}$ stands for the transposition of matrix $M$. Diagonal matrices are denoted by $\operatorname{diag}\{\cdot\}$. For a stochastic variable $\mathbf{x}(k)$, its probability and expectation are denoted by $\mathbb{P}\{\mathbf{x}(k)\}$ and $\mathbb{E}\{\mathbf{x}(k)\}$, respectively. $X>0$ stands for a positive definite matrix. $l_{2}[0, \infty)$ denotes the space of square summable infinite sequence. The term induced by symmetry is denoted by $*$.

\section{Problem Formulation}

2.1. System Description. In this paper, we consider the following fuzzy nonlinear system.

Plant Rule i. If $\delta_{1}(k)$ is $\xi_{i 1}, \delta_{2}(k)$ is $\xi_{i 2}$, and $\cdots$ and $\delta_{p}(k)$ is $\xi_{i p}$, then

$$
\begin{aligned}
\mathbf{x}(k+1) & =A_{i} \mathbf{x}(k)+B_{i} \mathbf{u}^{F}(k)+E_{i} \omega(k) \\
\mathbf{z}(k) & =C_{i} \mathbf{x}(k)+D_{i} \mathbf{u}^{F}(k)+F_{i} \omega(k) \\
\mathbf{y}(k) & =L_{i} \mathbf{x}(k)
\end{aligned}
$$

where $i \in \Theta=\{1,2, \ldots, r\}, r$ is the number of IF-THEN rules, $\xi_{i j}$ are the fuzzy sets, and $\delta_{1}(k), \delta_{2}(k), \ldots, \delta_{p}(k)$ are the premise variables. $\mathbf{x}(k) \in \mathbb{R}^{n}$ is the system state vector, $\mathbf{u}^{F}(k) \in \mathbb{R}^{m}$ is the control input vector, $\mathbf{z}(k) \in \mathbb{R}^{q}$ is the controlled output, and $\mathbf{y}(k) \in \mathbb{R}^{s}$ is the measurable output. $\omega(k) \in \mathbb{R}^{l}$ is the exogenous disturbance input which belongs to $l_{2}[0, \infty) . A_{i} \in \mathbb{R}^{n \times n}, B_{i} \in \mathbb{R}^{n \times m}, E_{i} \in \mathbb{R}^{n \times l}, C_{i} \in \mathbb{R}^{q \times n}, D_{i} \in$ $\mathbb{R}^{q \times m}, F_{i} \in \mathbb{R}^{q \times l}, L_{i} \in \mathbb{R}^{s \times n}$ are all constant real matrices with appropriate dimensions. Then, the resulting fuzzy nonlinear system can be inferred as follows:

$$
\begin{aligned}
\mathbf{x}(k+1) & =\sum_{i=1}^{r} h_{i}(\delta(k))\left\{A_{i} \mathbf{x}(k)+B_{i} \mathbf{u}^{F}(k)+E_{i} \omega(k)\right\} \\
\mathbf{z}(k) & =\sum_{i=1}^{r} h_{i}(\delta(k))\left\{C_{i} \mathbf{x}(k)+D_{i} \mathbf{u}^{F}(k)+F_{i} \omega(k)\right\} \\
\mathbf{y}(k) & =\sum_{i=1}^{r} h_{i}(\delta(k))\left\{L_{i} \mathbf{x}(k)\right\},
\end{aligned}
$$

where

$$
\begin{aligned}
& h_{i}(\delta(k))=\frac{\rho_{i}(\delta(k))}{\sum_{j=1}^{r} \rho_{j}(\delta(k))}, \\
& \rho_{i}(\delta(k))=\prod_{j=1}^{p} \xi_{i j}\left(\delta_{j}(k)\right),
\end{aligned}
$$

with $\xi_{i j}\left(\delta_{j}(k)\right)$ showing the grade of membership of $\delta_{j}(k)$ in $\xi_{i j}$. Then, it is not hard to see that $\rho_{i}(\delta(k)) \geq 0$ and $\sum_{j=1}^{r} \rho_{j}(\delta(k))>0, \forall k$, which implies $h_{i}(\delta(k)) \geq 0$, and $\sum_{i=1}^{r} h_{i}(\delta(k))=1, \forall k$. For the sake of brevity, in the sequel, we use $h_{i}$ and $h_{i}^{+}$to represent $h_{i}(\delta(k))$ and $h_{i}(\delta(k+1))$, respectively.

2.2. Actuator Faults. In this paper, we assume that there exist actuator faults and this phenomenon occurs randomly. Once the actuator failure occurs, the output of the actuator measurement values and the system input values are not consistent, namely $\mathbf{u}^{F}(k) \neq \mathbf{u}_{c}(k)$.

Considering the following actuator faults model, we use $\mathbf{u}^{F}(k)$ to represent the control signal that is sent to actuator:

$$
\mathbf{u}^{F}(k)=\alpha \mathbf{u}_{c}(k),
$$

where $\alpha=\operatorname{diag}\left\{\alpha_{1}, \alpha_{2}, \ldots, \alpha_{m}\right\}$, and

$$
0 \leq \underline{\alpha}_{l} \leq \alpha_{l} \leq \bar{\alpha}_{l} \leq 1, \quad l=1,2, \ldots, m \text {. }
$$

When $\underline{\alpha}_{l}=\bar{\alpha}_{l}=0$, it means that $l$ th actuator fails. When $0<\underline{\alpha}_{l}<\bar{\alpha}_{l}<1$, it implies that $l$ th actuator can only receive partial information. When $\underline{\alpha}_{l}=\bar{\alpha}_{l}=1$, it indicates that $l$ th actuator is in normal condition. Then, we define

$$
\begin{aligned}
& \widehat{\alpha}=\operatorname{diag}\left\{\frac{\bar{\alpha}_{1}+\underline{\alpha}_{1}}{2}, \frac{\bar{\alpha}_{2}+\underline{\alpha}_{2}}{2}, \ldots, \frac{\bar{\alpha}_{m}+\underline{\alpha}_{m}}{2}\right\} \\
& \check{\alpha}=\operatorname{diag}\left\{\frac{\bar{\alpha}_{1}-\underline{\alpha}_{1}}{2}, \frac{\bar{\alpha}_{2}-\underline{\alpha}_{2}}{2}, \ldots, \frac{\bar{\alpha}_{m}-\underline{\alpha}_{m}}{2}\right\} \\
& \delta_{l}=\operatorname{diag}\left\{\delta_{1}, \delta_{2}, \ldots, \delta_{m}\right\} .
\end{aligned}
$$


Then, it easy to obtain

$$
\alpha=\widehat{\alpha}+\delta_{l}
$$

where

$$
\left|\delta_{l}\right| \leq \frac{\bar{\alpha}_{l}-\underline{\alpha}_{l}}{2}, \quad l=1,2, \ldots, m
$$

Remark 1. In actual control systems, the actuator faults can occur inevitably which may be caused by the component natural aging and the harsh environment. Without considering, the actuator faults would degrade the system performance and even lead to unstable system.

2.3. Logarithmic Quantization. The logarithmic quantizer is described by

$$
\mathbf{y}_{q}(k)=Q(\mathbf{y}(k))
$$

where $Q(\mathbf{y}(k))=\left[\begin{array}{llll}Q_{1}\left(y_{1}(k)\right) & Q_{2}\left(y_{2}(k)\right) & \cdots & Q_{m}\left(y_{m}(k)\right)\end{array}\right]^{T}$, and the logarithmic quantizer is depicted by the following set of quantization levels:

$$
\begin{aligned}
U_{j}=\left\{ \pm \mu_{j i}: \mu_{j i}=\rho_{j}^{i} \mu_{0}, i=0, \pm 1,\right. & \pm 2, \ldots\} \cup\{0\}, \\
& 0<\rho_{j}<1, \mu_{0}>0,
\end{aligned}
$$

where parameter $\rho_{j}$ is the quantization density. For the logarithmic quantizer, associate quantizer $Q_{j}(\cdot)$ is defined by the following:

$$
\begin{aligned}
& Q_{j}\left(y_{j}(k)\right) \\
& = \begin{cases}\mu_{j i} & \text { if } \frac{1}{1+\varrho_{j}} \mu_{j i}<y_{j}(k) \leq \frac{1}{1-\varrho_{j}} \mu_{j i} \\
0 & \text { if } y_{j}(k)=0 \\
-Q_{j}\left(-y_{j}(k)\right) & \text { if } y_{j}(k)<0,\end{cases}
\end{aligned}
$$

where

$$
\varrho_{j}=\frac{1-\rho_{j}}{1+\rho_{j}} .
$$

Then, a sector bound expression can be described as follows:

$$
Q_{j}\left(y_{j}(k)\right)=\left(I+\Delta_{j}(k)\right) y_{j}(k), \quad\left|\Delta_{j}(k)\right| \leq \varrho_{j},
$$

where $\Delta_{j}(k) y_{j}(k)$ represents quantization error.

2.4. Dynamic Output Feedback Controller. In this subsection, the following dynamic output feedback controller is presented.

Controller Rule i. If $\delta_{1}(k)$ is $\xi_{i 1}, \delta_{2}(k)$ is $\xi_{i 2}$, and $\cdots$ and $\delta_{p}(k)$ is $\xi_{i p}$, then,

$$
\begin{aligned}
\mathbf{x}_{c}(k+1) & =A_{c i} \mathbf{x}_{c}(k)+B_{c i} \mathbf{y}_{c}(k) \\
\mathbf{u}_{c}(k) & =C_{c i} \mathbf{x}_{c}(k),
\end{aligned}
$$

where $\mathbf{x}_{c}(k) \in \mathbb{R}^{k}$ denotes the state vector of the controller; $\mathbf{u}_{c}(k) \in \mathbb{R}^{m}$ is the controller output; $\mathbf{y}_{c}(k) \in \mathbb{R}^{s}$ is the measurable output; $A_{c i}, B_{c i}$, and $C_{c i}$ are the controller gains to be designed. Then, the form of the controller can be described by

$$
\begin{aligned}
\mathbf{x}_{c}(k+1) & =\sum_{i=1}^{r} h_{i}(\delta(k))\left\{A_{c i} \mathbf{x}_{c}(k)+B_{c i} \mathbf{y}_{c}(k)\right\} \\
\mathbf{u}_{c}(k) & =\sum_{i=1}^{r} h_{i}(\delta(k))\left\{C_{c i} \mathbf{x}_{c}(k)\right\} .
\end{aligned}
$$

2.5. Closed-Loop System. Define $\zeta(k)=\left[\begin{array}{ll}\mathbf{x}(k)^{T} & \mathbf{x}_{c}(k)^{T}\end{array}\right]^{T}$, and combine (2), (4), (13), and (15); the following closed-loop system can be obtained:

$$
\begin{array}{r}
\zeta(k+1)=\sum_{i=1}^{r} \sum_{j=1}^{r} h_{i} h_{j}\left\{\widetilde{A}_{i j} \zeta(k)+\widetilde{E}_{i} \omega(k)\right\} \\
\mathbf{z}(k)=\sum_{i=1}^{r} \sum_{j=1}^{r} h_{i} h_{j}\left\{\widetilde{C}_{i j} \zeta(k)+\widetilde{F}_{i} \omega(k)\right\},
\end{array}
$$

where

$$
\begin{aligned}
\widetilde{A}_{i j} & =\left[\begin{array}{cc}
A_{i} & \alpha B_{i} C_{c j} \\
B_{c j}(I+\Delta(k)) L_{i} & A_{c j}
\end{array}\right] \\
\widetilde{C}_{i j} & =\left[\begin{array}{ll}
C_{i} & \alpha D_{i} C_{c j}
\end{array}\right] \\
\widetilde{E}_{i} & =\left[\begin{array}{ll}
E_{i}^{T} & 0
\end{array}\right]^{T} \\
\widetilde{F}_{i} & =F_{i} .
\end{aligned}
$$

\subsection{Main Objective}

Definition 2. The system in (16) is mean-square stable with $\omega(k)=0$, and if there exists finite $H>0$ such that the following inequality holds:

$$
\mathbb{E}\left\{\left.\sum_{k=0}^{\infty}\|\zeta(k)\|^{2}\right|_{\zeta(0)}\right\}<\zeta(0)^{T} H \zeta(0)
$$

for any initial condition $\zeta(0)$.

In order to gain the main results, the following lemma should be introduced.

Lemma 3 (see [30]). Given matrices $\Xi_{1}, \Xi_{2}$, and $\Xi_{3}$ of appropriate dimensions and with $\Xi_{1}$ symmetrical, then

$$
\Xi_{1}+\Xi_{3} \Delta(k) \Xi_{2}+\Xi_{2}^{T} \Delta(k)^{T} \Xi_{3}^{T}<0,
$$

holds for all $\Delta(k)$ satisfying $\Delta(k)^{T} \Delta(k) \leq I$, if and only, if there exists some $\epsilon>0$ such that

$$
\Xi_{1}+\epsilon \Xi_{2}^{T} \Xi_{2}+\epsilon^{-1} \Xi_{3} \Xi_{3}^{T}<0
$$


Now, we are in the position to state the problem of passive output feedback control for fuzzy nonlinear systems. For given scalar $\gamma>0$, design a dynamic output feedback controller (15) for systems in (2) such that the following two conditions simultaneously hold:

(a) Mean-square stability: the closed-loop system in (16) is mean-square stable with $\omega(k)=0$ and finite $H>0$ :

$$
\mathbb{E}\left\{\left.\sum_{k=0}^{\infty}\|\zeta(k)\|^{2}\right|_{\zeta(0)}\right\}<\zeta(0)^{T} H \zeta(0) \text {. }
$$

(b) Passive performance: for given scalar $\gamma$, under zero initial condition, the following inequality holds for all nonzero $\omega(k) \in l_{2}[0, \infty)$ :

$$
2 \mathbb{E}\left\{\sum_{k=0}^{\infty} \mathbf{z}(k)^{T} \omega(k)\right\} \geq-\gamma^{2} \mathbb{E}\left\{\sum_{k=0}^{\infty} \omega(k)^{T} \omega(k)\right\} .
$$

\section{Main Result}

In this section, we are going to research the mean-square stability and the passive performance of the system in (16); the corresponding result is presented in the following theorem.

Theorem 4. Given matrices $A_{c i}, B_{c i}$, and $C_{c i}$, scalars $\gamma>0$, the closed-loop system in (16) is mean-square stable and satisfies the passivity, if there exist matrices $P_{i}>0$ such that the inequalities hold:

$$
\left[\begin{array}{ccc}
-P_{i}^{-1} & -\widetilde{C}_{i j}^{T} & \widetilde{A}_{i j}^{T} \\
* & -\widetilde{F}_{i}^{T}-\widetilde{F}_{i}-\gamma^{2} I & \widetilde{E}_{i}^{T} \\
* & * & -P_{l}
\end{array}\right]<0,
$$

for all $i, j \in \Theta$.

Proof. In order to research the mean-square stability of the system (16) under condition $\omega(k)=0$, the following Lyapunov function is constructed:

$$
V(\zeta(k)) \triangleq \zeta(k)^{T}\left(\sum_{i=1}^{r} h_{i} P_{i}^{-1}\right) \zeta(k) .
$$

The difference of the Lyapunov function in (24) is defined as

$$
\mathbb{E}\{\Delta V(\zeta(k))\} \triangleq \mathbb{E}\left\{\left.V(\zeta(k+1))\right|_{\zeta(k)}\right\}-V(\zeta(k)) .
$$

Substituting (16) into (24), we have

$$
\begin{aligned}
\mathbb{E}\{\Delta V(\zeta(k))\} & \left\{\left.\zeta(k+1)^{T}\left(\sum_{k=1}^{r} h_{l}^{+} P_{l}^{-1}\right) \zeta(k+1)\right|_{\zeta(k)}\right\} \\
= & \mathbb{E}\left\{\zeta(k)^{T}\left(\sum_{i=1}^{r} h_{i} P_{i}^{-1}\right) \zeta(k)\right. \\
= & \sum_{l=1}^{r} \sum_{i=1}^{r} \sum_{j=1}^{r} \sum_{s=1}^{r} \sum_{t=1}^{r} h_{l}^{+} h_{i} h_{j} h_{s} h_{t} \zeta(k)^{T}\left\{\widetilde{A}_{i j}^{T} P_{l}^{-1} \widetilde{A}_{s t}-P_{i}^{-1}\right\} \zeta(k) .
\end{aligned}
$$

Define

$$
\begin{aligned}
\Omega_{l i j} & =\widetilde{A}_{i j}^{T} P_{l}^{-1} \widetilde{A}_{i j}-P_{i}^{-1} \\
\Omega(k) & =\sum_{i=1}^{r} \sum_{j=1}^{r} h_{i} h_{j} \Omega_{i j} .
\end{aligned}
$$

Then, we can conclude from (26) that $\mathbb{E}\{\Delta V(\zeta(k))\} \leq$ $\zeta(k)^{T} \Omega(k) \zeta(k)$, which means

$$
\mathbb{E}\left\{\left.V(\zeta(k+1))\right|_{\zeta(k)}\right\}-V(\zeta(k)) \leq \zeta(k)^{T} \Omega(k) \zeta(k) .
$$

Taking mathematical expectation and summing up the inequality on both sides from $k=0,1, \ldots, N$, we have

$$
\begin{aligned}
& \mathbb{E}\left\{\left.V(\zeta(k+1))\right|_{\zeta(0)}\right\}-V(\zeta(0)) \\
& \leq \mathbb{E}\left\{\sum_{k=0}^{N} \sum_{i=1}^{r} \sum_{j=1}^{r} h_{i} h_{j} \lambda_{\max }\left(\Omega_{i j}\right) \zeta(k)^{T} \zeta(k)\right\} .
\end{aligned}
$$

From the aforementioned inequalities, it is not hard to attain that, for $i, j=1, \ldots, r$, the following inequality holds:

$$
\begin{aligned}
\mathbb{E} & \left\{\sum_{k=0}^{N} \zeta(k)^{T} \zeta(k)\right\} \leq\left(\lambda_{\max }\left(\Omega_{i j}\right)\right)^{-1} \\
& \cdot\left\{V(\zeta(0))-\mathbb{E}\left\{\left.V(\zeta(k+1))\right|_{\zeta(0)}\right\}\right\} .
\end{aligned}
$$

Considering the nonzero initial condition and $\mathbb{E}\{V(\zeta(k))\} \geq$ 0 , as $N$ tends to infinity, from the above inequalities, we can conclude that

$$
\begin{aligned}
\mathbb{E}\left\{\sum_{k=0}^{\infty} \zeta(k)^{T} \zeta(k)\right\} & \leq\left(\lambda_{\max }\left(\Omega_{i j}\right)\right)^{-1} \zeta(0)^{T} P \zeta(0) \\
& =\psi \zeta(0)^{T} \zeta(0),
\end{aligned}
$$

where $\lambda_{\max }(\cdot)$ represents the maximum eigenvalues of $(\cdot)$, $\psi=\left(\lambda_{\max }\left(\Omega_{i j}\right)\right)^{-1} P, \forall i, j \in r$, and $\zeta(0)$ is the initial condition. According to Definition 2, the closed-loop system is meansquare stable.

Next, the passive performance for the system in (16) will be considered, under the zero initial conditions, and an index is introduced as follows:

$$
\mathbb{J}_{\mathbf{z} \omega}=\sum_{k=0}^{N-1} E\left\{-2 \mathbf{z}(k)^{T} \omega(k)-\gamma^{2} \omega(k)^{T} \omega(k)\right\}
$$


where $N$ is an arbitrary positive integer and nonzero $\omega(k) \epsilon$ $l_{2}[0, \infty)$. Similar to the previous derivation, we have

$$
\begin{aligned}
\mathbb{J}_{\mathbf{z} \omega} & =\sum_{k=1}^{N-1} \sum_{l=1}^{r} \sum_{i=1}^{r} \sum_{j=1}^{r} \sum_{s=1}^{r} \sum_{t=1}^{r} h_{l} h_{i} h_{j} h_{s} h_{t} \\
& \cdot\left\{\begin{array}{ll}
\zeta(k)^{T} & \omega(k)^{T}
\end{array}\right]\left[\begin{array}{c}
\widetilde{C}_{i j}^{T} \\
\widetilde{F}_{i}^{T}
\end{array}\right] \omega(k) \\
& +\left[\begin{array}{ll}
\zeta(k)^{T} & \omega(k)^{T}
\end{array}\right]\left[\begin{array}{c}
\widetilde{A}_{i j}^{T} \\
\widetilde{E}_{i}^{T}
\end{array}\right] P_{l}^{-1}\left[\begin{array}{ll}
\widetilde{A}_{s t} & \widetilde{E}_{s}
\end{array}\right]\left[\begin{array}{c}
\zeta(k) \\
\omega(k)
\end{array}\right] \\
& \left.-\zeta(k)^{T} P_{i}^{-1} f \zeta(k)-\gamma^{2} \omega(k)^{T} \omega(k)\right\} .
\end{aligned}
$$

By using Schur complement to (23), we infer that $\rrbracket_{z \omega} \leq 0$ for any $N$, which means the system in (16) satisfies the passive performance.

\section{Dynamic Output Feedback \\ Controller Design}

In this section, we aim to design the parameters of the controller in (15) based on Theorem 4, such that the closedloop system in (16) is mean-square stable and achieves the passive performance.

Theorem 5. Given scalars $\gamma>0$, the system in (16) satisfies the mean-square stability and achieves the passivity, if there exist symmetric positive definite matrices $R, S$, and $\widehat{A}_{c j}, \widehat{B}_{c j}, \widehat{C}_{c j}, V_{i}, J_{i}, K_{i}$, and scalar $\epsilon>0$ satisfying following conditions:

$$
\left[\begin{array}{ccccccccc}
\Gamma_{11} & \Gamma_{12}^{T} & \Gamma_{13} & \Gamma_{14}^{T} & 0 & \Gamma_{16}^{T} & 0 & \Gamma_{18}^{T} & 0 \\
* & \Gamma_{22} & \Gamma_{23} & 0 & 0 & 0 & 0 & 0 & -\epsilon D_{i} \\
* & * & \Gamma_{33} & 0 & \Gamma_{35}^{T} & 0 & \Gamma_{37}^{T} & 0 & 0 \\
* & * & * & -\epsilon I & 0 & 0 & 0 & 0 & 0 \\
* & * & * & * & -\epsilon I & 0 & 0 & 0 & 0 \\
* & * & * & * & * & -\epsilon I & 0 & 0 & 0 \\
* & * & * & * & * & * & -\epsilon I & 0 & 0 \\
* & * & * & * & * & * & * & -\epsilon I & 0 \\
* & * & * & * & * & * & * & * & -\epsilon I
\end{array}\right]<0,
$$

where

$$
\begin{aligned}
\Gamma_{11} & =\left[\begin{array}{cc}
V_{i}-R-R^{T} & J_{i}-2 I \\
* & K_{i}-S-S^{T}
\end{array}\right] \\
\Gamma_{12} & =\left[\begin{array}{ll}
-C_{i}-C_{i} S-\widehat{\alpha} D_{i} \widehat{C}_{c j}
\end{array}\right]
\end{aligned}
$$

$$
\begin{aligned}
& \Gamma_{13}=\left[\begin{array}{cc}
A_{i}^{T} R+L_{i}^{T} \widehat{B}_{c j}^{T} & A_{i}^{T} \\
\widehat{A}_{c j}^{T} & S^{T} A_{i}^{T}+\widehat{\alpha} C_{c j}^{T} B_{i}^{T}
\end{array}\right] \\
& \Gamma_{14}=\left[\begin{array}{ll}
\epsilon \varrho L_{i} & \epsilon \varrho L_{i} S
\end{array}\right] \\
& \Gamma_{16}=\Gamma_{18}=\left[\begin{array}{ll}
0 & \breve{\alpha} \widehat{C}_{c j}
\end{array}\right] \\
& \Gamma_{22}=-\widetilde{F}_{i}^{T}-\widetilde{F}_{i}-\gamma^{2} I \\
& \Gamma_{23}=\left[\begin{array}{ll}
E_{i}^{T} R & E_{i}^{T}
\end{array}\right] \\
& \Gamma_{33}=\left[\begin{array}{cc}
-V_{l} & -J_{l} \\
* & -K_{l}
\end{array}\right] \\
& \Gamma_{35}=\left[\begin{array}{ll}
\widehat{B}_{c j}^{T} & 0
\end{array}\right] \\
& \Gamma_{37}=\left[\begin{array}{ll}
\epsilon B_{i}^{T} R & \epsilon B_{i}^{T}
\end{array}\right] .
\end{aligned}
$$

The parameters of controller can be inferred by

$$
\begin{aligned}
\widehat{A}_{c j} & =R^{T} A_{i} S+\widehat{\alpha} R^{T} B_{i} \widehat{C}_{c j}+\widehat{B}_{c j} L_{i} S+M A_{c j} N^{T} \\
\widehat{B}_{c j} & =M B_{c j} \\
\widehat{C}_{c j} & =C_{c j} N^{T} .
\end{aligned}
$$

Proof. In order to remove uncertain term $\Delta(k)$, the inequalities (22) can be rewritten to the following form:

$$
\begin{aligned}
& {\left[\begin{array}{ccc}
-P_{i}^{-1} & -\bar{C}_{i j}^{T} & \bar{A}_{i j}^{T} \\
* & -\widetilde{F}_{i}^{T}-\widetilde{F}_{i}-\gamma^{2} I & \widetilde{E}_{i}^{T} \\
* & * & -P_{l}
\end{array}\right]+X_{i}^{T} \frac{\Delta(k)^{T}}{\varrho} Y_{j}^{T}} \\
& \quad+Y_{j} \frac{\Delta(k)}{\varrho} X_{i}+N_{j}^{T} \Delta(k)^{T} M_{i}^{T}+M_{i} \Delta(k) N_{j} \\
& \quad+U_{j}^{T} \Delta(k)^{T} V_{i}^{T}+V_{i} \Delta(k) U_{j}<0,
\end{aligned}
$$

where

$$
\begin{aligned}
\bar{A}_{i j} & =\left[\begin{array}{cc}
A_{i} & \widehat{\alpha} B_{i} C_{c j} \\
B_{c j} L_{i} & A_{c j}
\end{array}\right] \\
\bar{C}_{i j} & =\left[\begin{array}{ll}
C_{i} & \widehat{\alpha} D_{i} C_{c j}
\end{array}\right] \\
X_{i} & =\left[\begin{array}{lll}
\varrho \widetilde{L}_{i} & 0 & 0
\end{array}\right] \\
Y_{j} & =\left[\begin{array}{lll}
0 & 0 & \widetilde{B}_{c j}^{T}
\end{array}\right]^{T} \\
M_{i} & =\left[\begin{array}{lll}
0 & 0 & \widetilde{B}_{i}^{T}
\end{array}\right]^{T}
\end{aligned}
$$




$$
\begin{aligned}
N_{j} & =\left[\begin{array}{lll}
\widetilde{C}_{c j} & 0 & 0
\end{array}\right] \\
V_{i} & =\left[\begin{array}{lll}
0 & -D_{i}^{T} & 0
\end{array}\right]^{T} \\
U_{j} & =\left[\begin{array}{lll}
\widetilde{C}_{c j} & 0 & 0
\end{array}\right] \\
\widetilde{C}_{c j} & =\left[\begin{array}{ll}
0 & \check{\alpha} C_{c j}
\end{array}\right]
\end{aligned}
$$

$$
\begin{aligned}
\widetilde{L}_{i} & =\left[\begin{array}{ll}
L_{i} & 0
\end{array}\right] \\
\widetilde{B}_{c j} & =\left[\begin{array}{ll}
0 & B_{c j}^{T}
\end{array}\right]^{T} \\
\widetilde{B}_{i} & =\left[\begin{array}{ll}
B_{i}^{T} & 0
\end{array}\right]^{T} .
\end{aligned}
$$

Using Schur complement and Lemma 3, we can obtain the inequalities as follows:

$$
\left[\begin{array}{ccccccccc}
-P_{i}^{-1} & -\bar{C}_{i j}^{T} & \bar{A}_{i j}^{T} & \epsilon \varrho \widetilde{L}_{i}^{T} & 0 & \widetilde{C}_{c j}^{T} & 0 & \widetilde{C}_{c j}^{T} & 0 \\
* & -\widetilde{F}_{i}^{T}-\widetilde{F}_{i}-\gamma^{2} I & \widetilde{E}_{i}^{T} & 0 & 0 & 0 & 0 & 0 & -\epsilon D_{i} \\
* & * & -P_{l} & 0 & \widetilde{B}_{c j} & 0 & \epsilon \widetilde{B}_{i} & 0 & 0 \\
* & * & * & -\epsilon I & 0 & 0 & 0 & 0 & 0 \\
* & * & * & * & -\epsilon I & 0 & 0 & 0 & 0 \\
* & * & * & * & * & -\epsilon I & 0 & 0 & 0 \\
* & * & * & * & * & * & -\epsilon I & 0 & 0 \\
* & * & * & * & * & * & * & -\epsilon I & 0 \\
* & * & * & * & * & * & * & * & -\epsilon I
\end{array}\right]<0 .
$$

It is not hard to see that there are no uncertain terms, and we need to eliminate nonlinearities in order to get parameters of the controller by Matlab LIM Toolbox. Consider the following inequality:

$$
\left(P_{i}-Q\right)^{T} P_{i}^{-1}\left(P_{i}-Q\right) \geq 0
$$

It implies that

$$
Q^{T} P_{i}^{-1} Q \geq Q+Q^{T}-P_{i}
$$

Define partition matrices $Q, Q^{-1}$ as follows:

$$
\begin{aligned}
Q & =\left[\begin{array}{cc}
S & N \\
N^{T} & W
\end{array}\right] \\
Q^{-1} & =\left[\begin{array}{cc}
R & M \\
M^{T} & U
\end{array}\right],
\end{aligned}
$$

where $Q, Q^{-1}, M, N \in \mathbb{R}^{n}$.

Then, we can conclude from equation $Q Q^{-1}=I$ that

$$
M N^{T}=I-R S .
$$

Suppose that inequalities (34) and (35) hold, then nonsingular matrices $R$ and $S$ can be obtained, which means that matrices $I-R S$ are also nonsingular, then the nonsingular matrices $M$ and $N$ can be inferred by (44).

Define

$$
\begin{aligned}
& H_{1}=\left[\begin{array}{cc}
R & I \\
M^{T} & 0
\end{array}\right], \\
& H_{2}=\left[\begin{array}{cc}
I & S \\
0 & N^{T}
\end{array}\right] .
\end{aligned}
$$

By simple calculation, we can get

$$
\begin{aligned}
Q H_{1} & =H_{2}, \\
H_{1}^{T} Q H_{1} & =H_{1}^{T} H_{2}=\left[\begin{array}{ll}
R & I \\
I & S
\end{array}\right] \\
P_{i} & =H_{1}^{-T}\left[\begin{array}{ll}
V_{i} & J_{i} \\
* & K_{i}
\end{array}\right] H_{1}^{-1} .
\end{aligned}
$$


Based on the partition matrices defined above, then premultiplying and postmultiplying inequalities (34) with $\operatorname{diag}\left\{H_{1}^{-T}\right.$,
$\left.I, H_{1}^{-T}, I, I, I, I, I, I\right\}$ and $\operatorname{diag}\left\{H_{1}^{-1}, I, H_{1}^{-1}, I, I, I, I, I, I\right\}$ respectively, we can get

$$
\left[\begin{array}{ccccccccc}
P_{i}-Q-Q^{T} & -Q^{T} \bar{C}_{i j}^{T} & Q^{T} \bar{A}_{i j}^{T} & Q^{T} \epsilon \widetilde{L}_{i}^{T} & 0 & Q^{T} \widetilde{C}_{c j}^{T} & 0 & Q^{T} \widetilde{C}_{c j}^{T} & 0 \\
* & -\widetilde{F}_{i}^{T}-\widetilde{F}_{i}-\gamma^{2} I & \widetilde{E}_{i}^{T} & 0 & 0 & 0 & 0 & 0 & -\epsilon D_{i} \\
* & * & -P_{l} & 0 & \widetilde{B}_{c j} & 0 & \epsilon \widetilde{B}_{i} & 0 & 0 \\
* & * & * & -\epsilon I & 0 & 0 & 0 & 0 & 0 \\
* & * & * & * & -\epsilon I & 0 & 0 & 0 & 0 \\
* & * & * & * & * & -\epsilon I & 0 & 0 & 0 \\
* & * & * & * & * & * & -\epsilon I & 0 & 0 \\
* & * & * & * & * & * & * & -\epsilon I & 0 \\
* & * & * & * & * & * & * & * & -\epsilon I
\end{array}\right]<0 .
$$

Employing inequalities (42) and (47), we can obtain

$$
\left[\begin{array}{ccccccccc}
-Q^{T} P_{i}^{-1} Q & -Q^{T} \bar{C}_{i j}^{T} & Q^{T} \bar{A}_{i j}^{T} & Q^{T} \epsilon \widetilde{L}_{i}^{T} & 0 & Q^{T} \widetilde{C}_{c j}^{T} & 0 & Q^{T} \widetilde{C}_{c j}^{T} & 0 \\
* & -\widetilde{F}_{i}^{T}-\widetilde{F}_{i}-\gamma^{2} I & \widetilde{E}_{i}^{T} & 0 & 0 & 0 & 0 & 0 & -\epsilon D_{i} \\
* & * & -P_{l} & 0 & \widetilde{B}_{c j} & 0 & \epsilon \widetilde{B}_{i} & 0 & 0 \\
* & * & * & -\epsilon I & 0 & 0 & 0 & 0 & 0 \\
* & * & * & * & -\epsilon I & 0 & 0 & 0 & 0 \\
* & * & * & * & * & -\epsilon I & 0 & 0 & 0 \\
* & * & * & * & * & * & -\epsilon I & 0 & 0 \\
* & * & * & * & * & * & * & -\epsilon I & 0 \\
* & * & * & * & * & * & * & * & -\epsilon I
\end{array}\right]<0 .
$$

Then, performing congruence transformation to (48) with $\operatorname{diag}\left\{Q^{-1}, I, I, I, I, I, I, I, I\right\}$, we get (40).

We can conclude that if inequalities (34) and (35) hold, then we can guarantee that inequalities (40) meet the condition, which implies that inequalities (22) hold. In short, if inequalities (34) and (35) hold, then the closed system in (16) is mean-square stability with the guaranteed passivity.

\section{Numerical Example}

In this section, the results will be demonstrated by the following example for system (2) which is expressed by $T-S$ fuzzy model with two IF-THEN rules $(r=2)$.

Rule 1.

$$
A_{1}=\left[\begin{array}{ccc}
0.3 & 0.6 & 0.8 \\
-0.2 & 0.7 & 0.1 \\
0.25 & 0 & 0.6
\end{array}\right]
$$

$$
\begin{aligned}
B_{1} & =\left[\begin{array}{lll}
0.7 & 0 & 0
\end{array}\right]^{T} \\
C_{1} & =\left[\begin{array}{lll}
-0.1 & -0.1 & 0.3
\end{array}\right] \\
E_{1} & =\left[\begin{array}{lll}
0.001 & 0.003 & 0.006
\end{array}\right]^{T} \\
D_{1} & =0.4 \\
F_{1} & =0.001 \\
L_{1} & =\left[\begin{array}{ccc}
1 & 1 & 1 \\
0.1 & 0.3 & -0.6
\end{array}\right] .
\end{aligned}
$$

Rule 2.

$$
\begin{aligned}
& A_{2}=\left[\begin{array}{ccc}
0.6 & 0.8 & -0.9 \\
0.3 & 0.6 & 0 \\
-0.6 & 0.4 & 0.7
\end{array}\right] \\
& B_{2}=\left[\begin{array}{lll}
0.5 & 0 & 0
\end{array}\right]^{T}
\end{aligned}
$$


TABLE 1: $\gamma^{2}$ with different actuator faults.

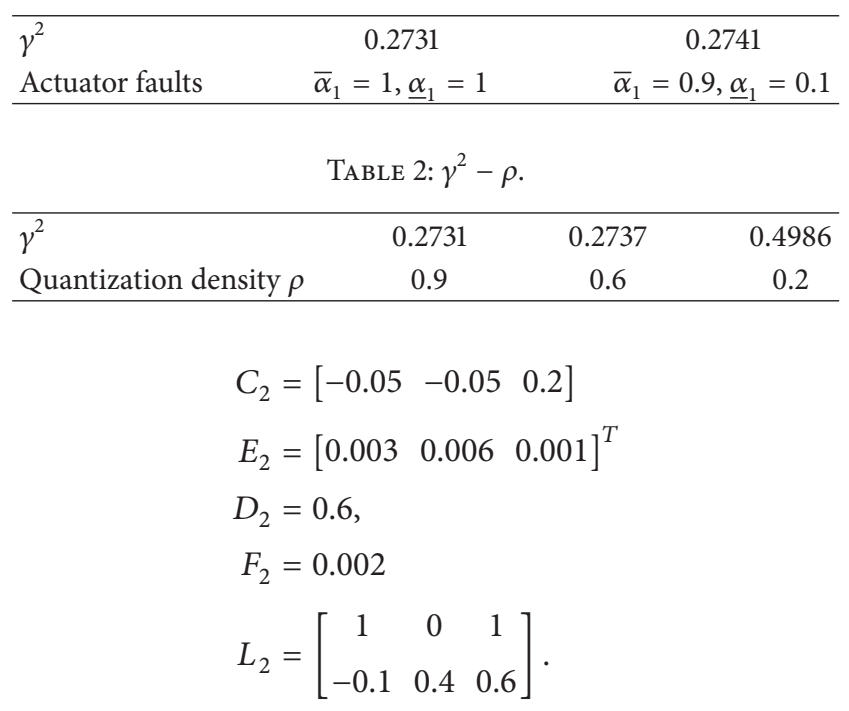

First, considering the quantization parameters assumed to be $\varrho_{1}=\varrho_{2}=0.05$, we study the effect of different actuator failure model on the system passive performance, which are presented clearly in Table 1 . From Table 1, we can see that, when the actuator failures increase, the system passive performance will degrade.

Then, we consider the influence of different quantization density on the system passive performance under conditions $\bar{\alpha}_{1}=0.9, \underline{\alpha}_{1}=0.6$. From Table 2 , we can conclude that the system performance will decrease when the quantization density reduces.

Now, we assume that the quantization parameters are $\varrho_{1}=\varrho_{2}=0.05$ and the actuator failures are $\bar{\alpha}_{1}=0.9$, $\underline{\alpha}_{1}=0.6 . \epsilon$ is chosen to be $\epsilon=1$. According to Theorem 5 and using the LMI Toolbox, we obtain the minimum of passive performance $\gamma^{2}=0.2731$ and the corresponding output feedback controller parameters are as follows:

$$
\begin{aligned}
& A_{c 1}=\left[\begin{array}{ccc}
0.0694 & 1.0686 & -11.4408 \\
0.0600 & -0.7322 & -0.6388 \\
-0.2004 & 2.9238 & -2.0978
\end{array}\right] \\
& B_{c 1}=\left[\begin{array}{lll}
0.0596 & -4.0088 & 11.4672 \\
0.4984 & -0.5796 & 1.6838
\end{array}\right]^{T} \\
& C_{c 1}=\left[\begin{array}{lll}
-0.0070 & 0.0037 & 1.5097
\end{array}\right] \\
& A_{c 2}=\left[\begin{array}{ccc}
0.2180 & -1.1328 & 8.6790 \\
-0.0508 & 0.8610 & 1.4932 \\
0.2198 & -3.1868 & 1.1138
\end{array}\right]^{-1.13} \\
& B_{c 2}=\left[\begin{array}{lll}
-0.8860 & 3.7404 & -11.4998 \\
0.1464 & 0.2686 & -1.8178
\end{array}\right]^{T} \\
& C_{c 2}=\left[\begin{array}{lll}
0.0002 & 0.1105 & 5.5350
\end{array}\right] .
\end{aligned}
$$

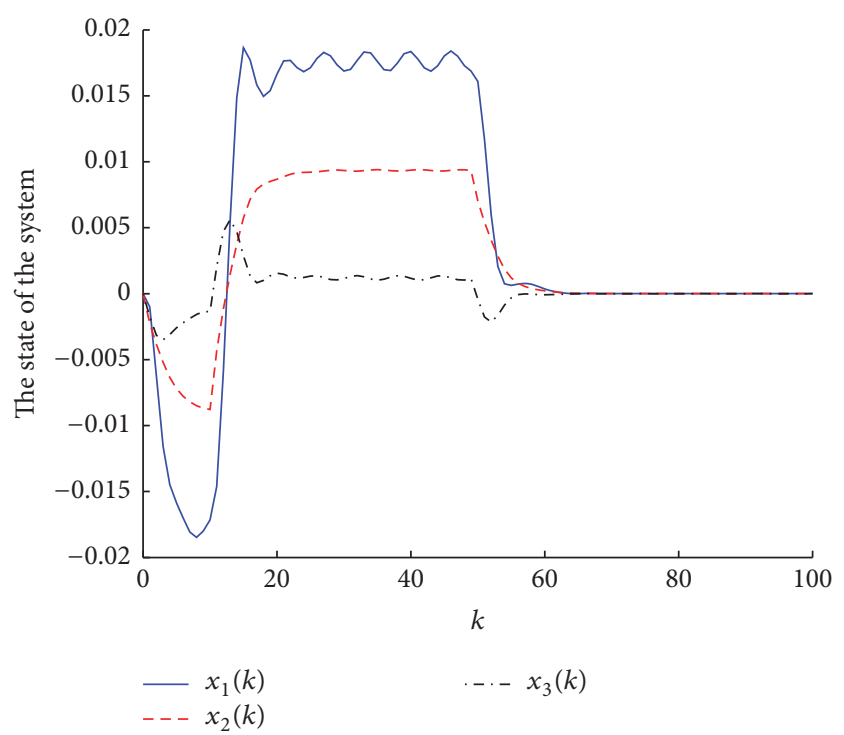

Figure 1: The state response of $\mathbf{x}(k)$.

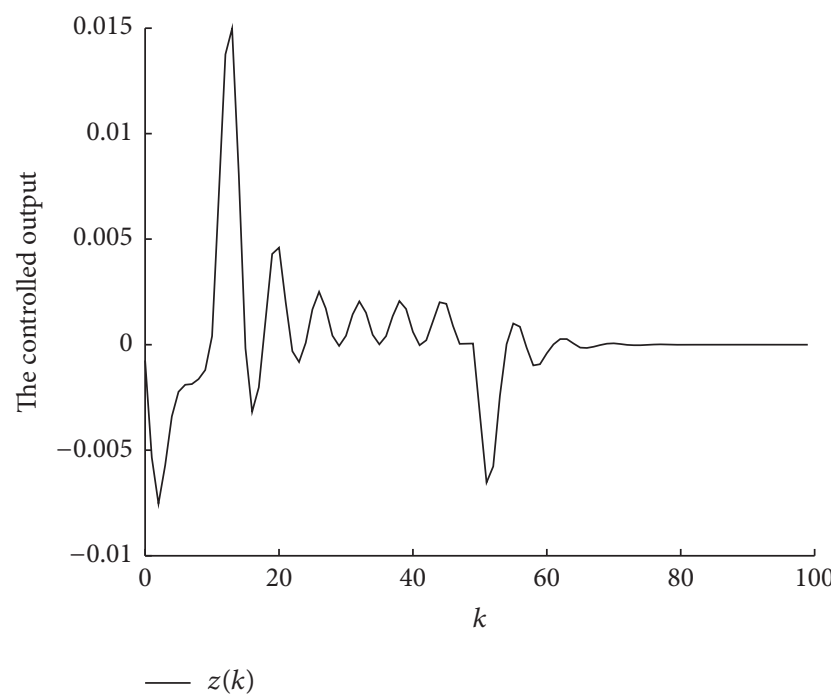

Figure 2: Controlled output $\mathbf{z}(k)$.

The disturbance noise is

$$
\nu(k)= \begin{cases}-0.5 & 0 \leq k \leq 10 \\ 0.5 & 11 \leq k \leq 50 \\ 0 & 51 \leq k \leq 60 .\end{cases}
$$

Then, we can obtain the trajectory of the states which are shown in Figure 1. Figure 2 demonstrates the controlled output of the closed-loop system, and Figure 3 depicts the state response of $\mathbf{x}_{c}(k)$ in the closed-loop system.

\section{Conclusion}

In this paper, we have investigated the passive dynamic output feedback control of the fuzzy discrete nonlinear 


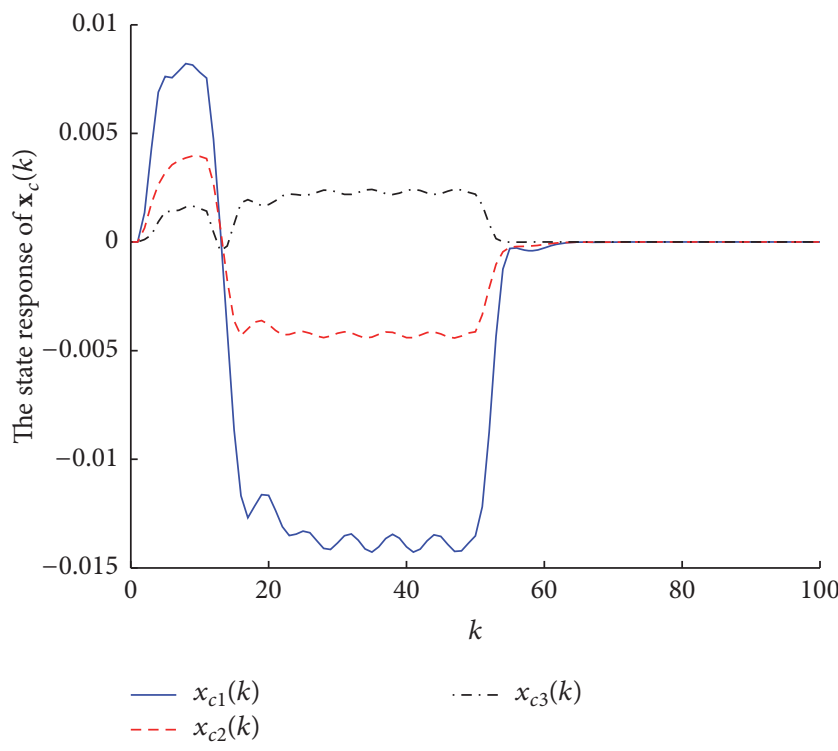

Figure 3: The state response of $\mathbf{x}_{c}(k)$.

system with quantization and actuator failures, where the measurable output is quantized by a logarithmic quantizer. The robust passive dynamic output feedback controller has been proposed to deal with the effects of the actuator failures and mitigate quantization effects. Sufficient condition has been obtained to guarantee that the closed-loop system is mean-square stable and achieves the passive performance. A numerical example has been provided to show the effectiveness of the proposed approach.

\section{Competing Interests}

The authors declare that they have no competing interests.

\section{Acknowledgments}

This work was supported by the National Natural Science Foundation of China under Grants 61573136, 61603133, and 61503136 and the Science Technology Department of Zhejiang Province in China under Grant 2014C33004.

\section{References}

[1] T. Takagi and M. Sugeno, "Fuzzy identification of systems and its applications to modeling and control," IEEE Transactions on Systems, Man and Cybernetics, vol. 15, no. 1, pp. 116-132, 1985.

[2] G. Feng, "A survey on analysis and design of model-based fuzzy control systems," IEEE Transactions on Fuzzy Systems, vol. 14, no. 5, pp. 676-697, 2006.

[3] H. Li, C. Wu, X. Jing, and L. Wu, "Fuzzy tracking control for nonlinear networked systems," IEEE Transactions on Cybernetics, 2016.

[4] Q. Zhou, H. Li, C. Wu, L. Wang, and C. K. Ahn, "Adaptive fuzzy control of nonlinear systems with unmodeled dynamics and input saturation using small-gain approach," IEEE Transactions on Systems, Man and Cybernetics: Systems, 2016.
[5] L. Zhao, H. Gao, and H. R. Karimi, "Robust stability and stabilization of uncertain T-S fuzzy systems with time-varying delay: an input-output approach," IEEE Transactions on Fuzzy Systems, vol. 21, no. 5, pp. 883-897, 2013.

[6] H. Gao, X. Liu, and J. Lam, "Stability analysis and stabilization for discrete-time fuzzy systems with time-varying delay," IEEE Transactions on Systems, Man, and Cybernetics, Part B: Cybernetics, vol. 39, no. 2, pp. 306-317, 2009.

[7] Y. Zhao, H. Gao, J. Lam, and B. Du, "Stability and stabilization of delayed T-S fuzzy systems: a delay partitioning approach," IEEE Transactions on Fuzzy Systems, vol. 17, no. 4, pp. 750-762, 2009.

[8] H. Gao and T. Chen, "Stabilization of nonlinear systems under variable sampling: a fuzzy control approach," IEEE Transactions on Fuzzy Systems, vol. 15, no. 5, pp. 972-983, 2007.

[9] H. Gao, Y. Zhao, and T. Chen, " $H_{\infty}$ fuzzy control of nonlinear systems under unreliable communication links," IEEE Transactions on Fuzzy Systems, vol. 17, no. 2, pp. 265-278, 2009.

[10] Y. Zhao and H. Gao, "Fuzzy-model-based control of an overhead crane with input delay and actuator saturation," IEEE Transactions on Fuzzy Systems, vol. 20, no. 1, pp. 181-186, 2012.

[11] J. Qiu, G. Feng, and H. Gao, "Fuzzy-model-basedpiecewise $H_{\infty}$ static-output-feedback controller design for networked nonlinear systems," IEEE Transactions on Fuzzy Systems, vol. 18, no. 5, pp. 919-934, 2010.

[12] H. Gao, Y. Zhao, J. Lam, and K. Chen, " $H_{\infty}$ fuzzy filtering of nonlinear systems with intermittent measurements," IEEE Transactions on Fuzzy Systems, vol. 17, no. 2, pp. 291-300, 2009.

[13] X. Su, L. Wu, and P. Shi, "Sensor networks with random link failures: distributed filtering for T-S fuzzy systems," IEEE Transactions on Industrial Informatics, vol. 9, no. 3, pp. 17391750, 2013.

[14] Y. Niu, D. W. C. Ho, and C. W. Li, "Filtering for discrete fuzzy stochastic systems with sensor nonlinearities," IEEE Transactions on Fuzzy Systems, vol. 18, no. 5, pp. 971-978, 2010.

[15] X. Su, P. Shi, L. Wu, and M. V. Basin, "Reliable filtering with strict dissipativity for T-S fuzzy time-delay systems," IEEE Transactions on Cybernetics, vol. 44, no. 12, pp. 2470-2483, 2014.

[16] H. Li, Y. Gao, L. Wu, and H. K. Lam, "Fault detection for T$S$ fuzzy time-delay systems: delta operator and input-output methods," IEEE Transactions on Cybernetics, vol. 45, no. 2, pp. 229-241, 2015.

[17] M. Liu, P. Shi, L. Zhang, and X. Zhao, "Fault-tolerant control for nonlinear Markovian jump systems via proportional and derivative sliding mode observer technique," IEEE Transactions on Circuits and Systems. I. Regular Papers, vol. 58, no. 11, pp. 2755-2764, 2011.

[18] Z. Feng and J. Lam, "Robust reliable dissipative filtering for discrete delay singular systems," Signal Processing, vol. 92, no. 12, pp. 3010-3025, 2012.

[19] Z. Feng, W. X. Zheng, and L. Wu, "Reachable set estimation of T-S fuzzy systems with time-varying delay," IEEE Transactions on Fuzzy Systems, 2016.

[20] D. Zhang, W. Zhang, L. Yu, and Q.-G. Wang, "Distributed fault detection for a class of large-scale systems with multiple incomplete measurements," Journal of the Franklin Institute, vol. 352, no. 9, pp. 3730-3749, 2015.

[21] J. P. Hespanha, P. Naghshtabrizi, and Y. Xu, "A survey of recent results in networked control systems," Proceedings of the IEEE, vol. 95, no. 1, pp. 138-172, 2007.

[22] R. Lu, Y. Xu, A. Xue, and J. Zheng, "Networked control with state reset and quantized measurements: observer-based case," 
IEEE Transactions on Industrial Electronics, vol. 60, no. 11, pp. 5206-5213, 2013.

[23] Y. Xu, H. Su, Y.-J. Pan, Z.-G. Wu, and W. Xu, "Stability analysis of networked control systems with round-robin scheduling and packet dropouts," Journal of the Franklin Institute. Engineering and Applied Mathematics, vol. 350, no. 8, pp. 2013-2027, 2013.

[24] L. Zhang, H. Gao, and O. Kaynak, "Network-induced constraints in networked control systems: a survey," IEEE Transactions on Industrial Informatics, vol. 9, no. 1, pp. 403-416, 2013.

[25] H. Dong, Z. Wang, and H. Gao, " $H_{\infty}$ fuzzy control for systems with repeated scalar nonlinearities and random packet losses," IEEE Transactions on Fuzzy Systems, vol. 17, no. 2, pp. 440-450, 2009.

[26] Z. Wang, F. Yang, D. W. C. Ho, and X. Liu, "Robust $H_{\infty}$ control for networked systems with random packet losses," IEEE Transactions on Systems, Man, and Cybernetics, Part B: Cybernetics, vol. 37, no. 4, pp. 916-924, 2007.

[27] G. Wei, G. Feng, and Z. Wang, "Robust $H_{\infty}$ control for discrete-time fuzzy systems with infinite-distributed delays," IEEE Transactions on Fuzzy Systems, vol. 17, no. 1, pp. 224-232, 2009.

[28] S. K. Nguang and P. Shi, "Fuzzy output feedback control design for nonlinear systems: an LMI approach," IEEE Transactions on Fuzzy Systems, vol. 11, no. 3, pp. 712-725, 2003.

[29] H. Li, C. Wu, and Z. Feng, "Fuzzy dynamic output-feedback control of non-linear networked discrete-time system with missing measurements," IET Control Theory and Applications, vol. 9, no. 3, pp. 327-335, 2015.

[30] L. Xie, "Output feedback $H_{\infty}$ control of systems with parameter uncertainty," International Journal of Control, vol. 63, no. 4, pp. 741-750, 1996. 


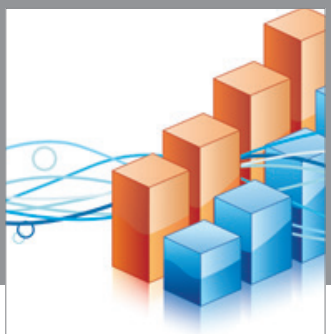

Advances in

Operations Research

vatem alat4

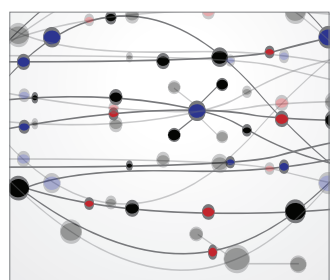

\section{The Scientific} World Journal
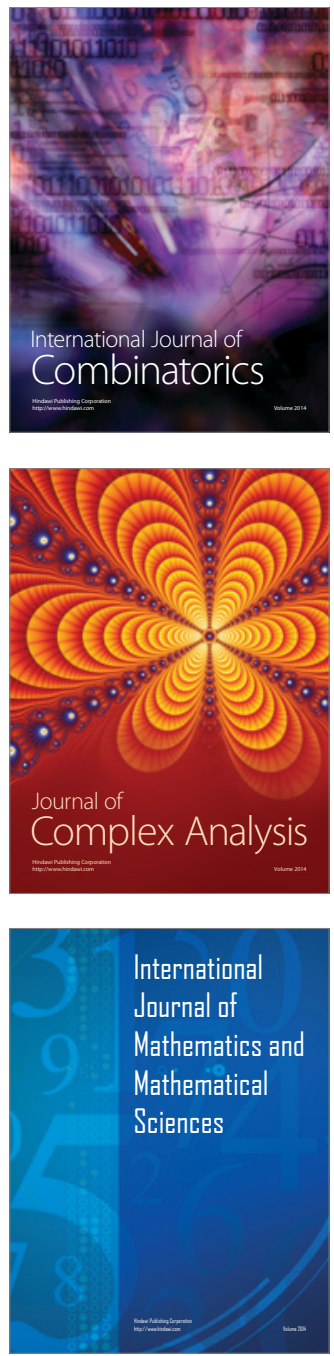
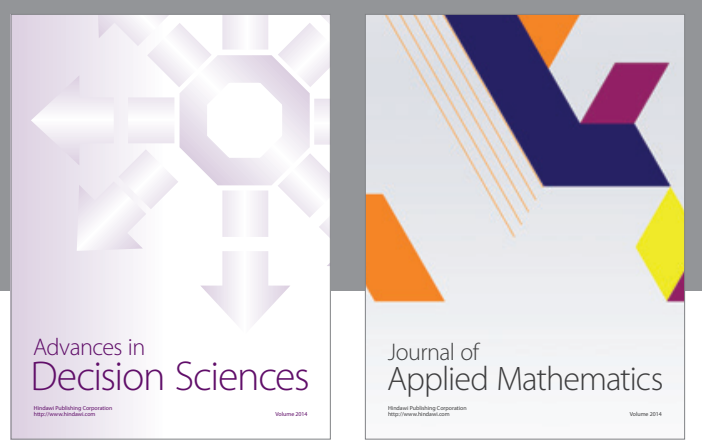

Algebra

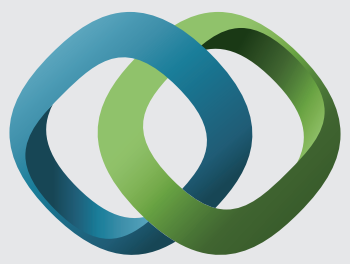

\section{Hindawi}

Submit your manuscripts at

http://www.hindawi.com
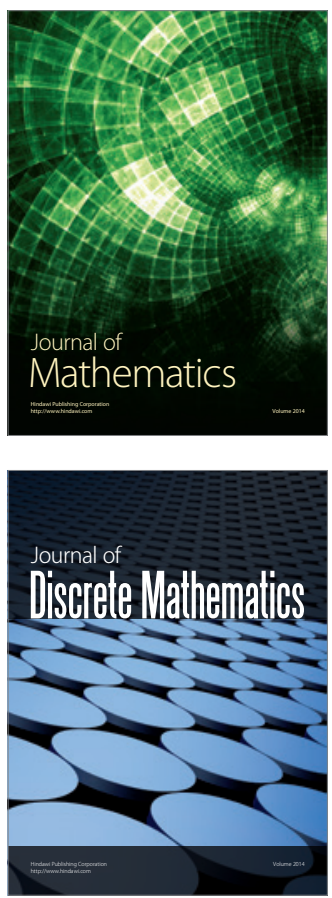

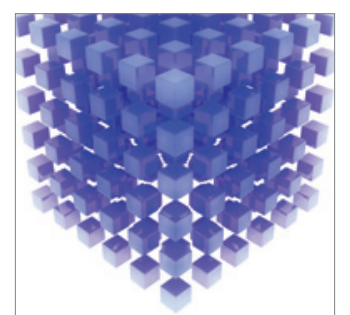

Mathematical Problems in Engineering
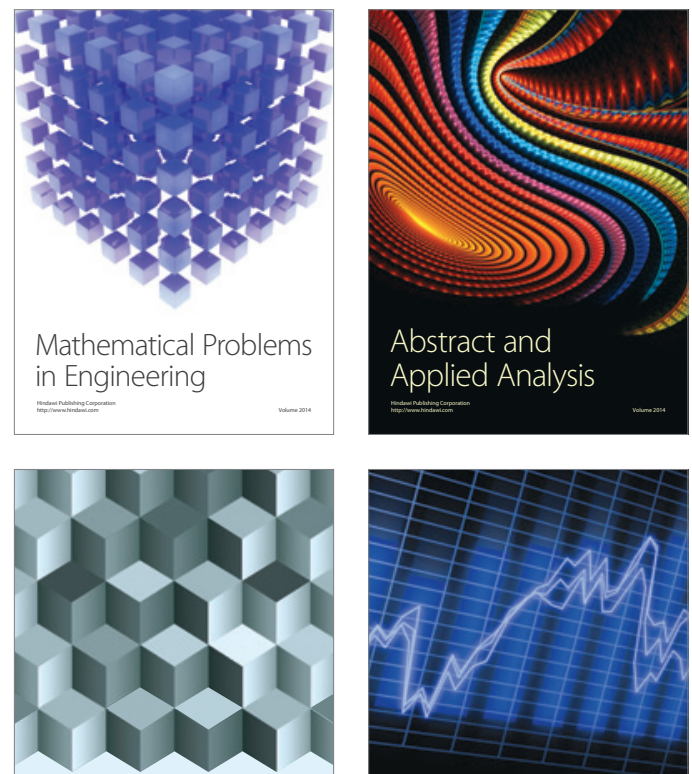

Journal of

Function Spaces

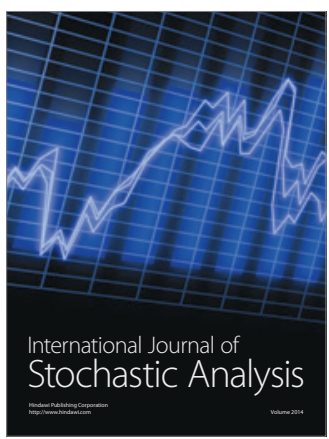

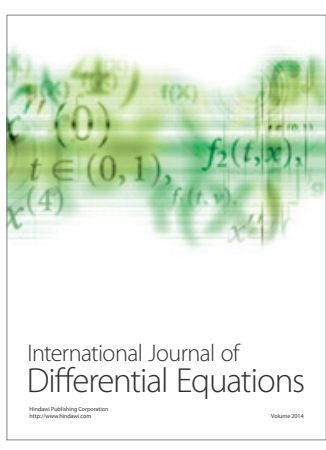
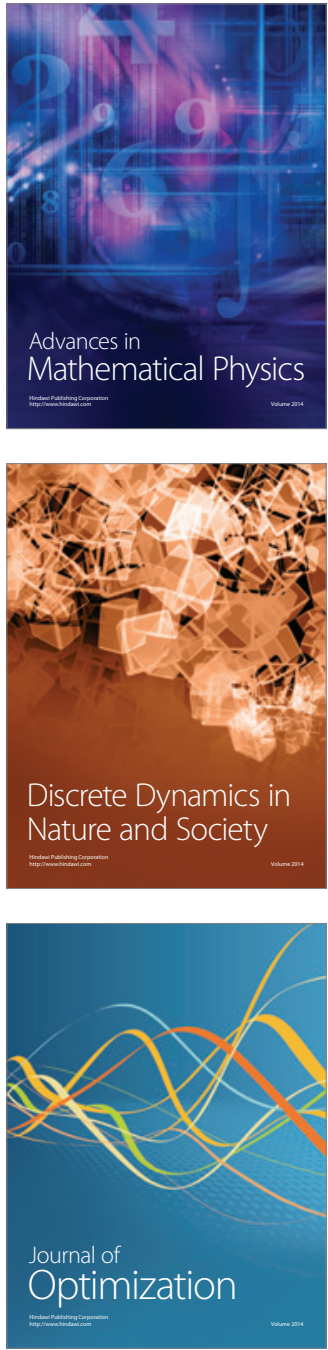